\title{
Tandem-L Observation Concept - Contributions and Challenges of Systematic Monitoring of Earth System Dynamics
}

\author{
Daniela Borla Tridon ${ }^{1}$, Markus Bachmann ${ }^{1}$, Francesco De Zan $^{2}$, Gerhard Krieger ${ }^{1}$, \\ Manfred Zink $^{1}$, Daniel Schulze ${ }^{1}$, Alberto Moreira ${ }^{1}$ \\ German Aerospace Center (DLR) \\ ${ }^{1}$ Microwaves and Radar Institute, ${ }^{2}$ Remote Sensing Technology Institute \\ Oberpfaffenhofen, Germany \\ email: daniela.borlatridon@dlr.de
}

\begin{abstract}
Tandem-L is a proposal for a spaceborne L-band SAR mission that aims at the global monitoring of dynamic processes on the Earth. The mission will acquire an enormous amount of data for different applications. These data will be used to provide information concerning biosphere, geosphere, cryosphere, and hydrosphere. Within the scope of this mission, an observation concept has to be developed in order to fulfill all the scientific requirements and clarify the necessary system resources. A sophisticated planning and optimization process has been implemented. It combines the predetermined satellite resources, ground station network, and the scientific requirements to generate an acquisition timeline on individual data take level. The resulting timeline fulfills all requirements for the various scientific applications and can be used to analyze different aspects of the mission, e.g. data volumes and orbit usage are necessary to refine the on-board and ground resources.
\end{abstract}

\section{Introduction}

Global monitoring of environmental information is essential in order to understand dynamics of different Earth system processes in the geosphere, biosphere, cryosphere, and hydrosphere [1]. To this scope, a proposal for a unique spaceborne SAR mission, called Tandem-L, has been elaborated. To satisfy the challenging scientific user demands, the Tandem-L mission concept has been developed based on the use of two SAR satellites operating in L-band $(23.6 \mathrm{~cm}$ wavelength) each equipped with a reflector antenna and exploiting the digital beam forming technique [2], [3]. This will allow the system to generate high-resolution wide-swath images and to increase the coverage. The two satellites will be able to fly in different formation flight configurations, and to acquire data with different polarizations, depending on the requirements for the various scientific research fields. The project is currently in a phase B. Assuming the approval for full implementation in 2017, the Tandem-L satellites could be launched around 2022/23.

The choice of L-band is driven by main requirements for tomographic measurements of the 3-D structure of vegetation and ice, thanks to high signal penetration, and for the long-term measurements of deformations with millimeter accuracy, thanks to the high correlation. 
Based on the scientific user requirements, a set of 24 bio/geophysical products has been defined. In order to fulfill all the requirements, a satisfactory observation concept needs to be developed [4].

It has to accommodate all the requirements in term of spatial and temporal coverage, swath width, resolution, polarization, interferometric operation and perpendicular baselines. Initial constraints are the instrument capabilities, downlink capacity, on-board memory and orbit usage. The derived scenario, which is expressed in terms of an acquisition timeline, will then in turn help to refine the necessary resources of the mission. One of the main challenges in designing the observation concept is to combine requirements from different applications which might not be compatible to each other. As the project is in an early phase, another challenge is also the relatively high variability of the requirements and priority of the individual applications and their constraints. The observation concept has to react to these changes with frequent updates of the acquisition timeline.

Tandem- $\mathrm{L}$ is based on the experience gained from the missions TerraSAR-X and TanDEM-X. TanDEM-X acquired a global digital elevation model using two SAR satellites in X-band, flying in a controlled formation [5], [6]. The great success of the mission proves the potential of such a satellite formation and acquisition concept. Because of the higher complexity of the Tandem-L mission, this concept has been further developed.

The aim of this paper is then to describe the observation concept developed for the Tandem- $\mathrm{L}$ mission with its major challenges and constraints.

\section{Acquisitions concept}

\subsection{Acquisitions requirements}

In order to generate an optimal observation concept, the requirements for each scientific application have to be pre-analyzed and combined together. The requirements might differ from each other depending on the resolution needed for the different applications. This in turn affects the way the acquisitions are planned. In details, acquisition modes differ by polarization, swath width and bandwidth, as listed in Table 1. Moreover, all these modes have the noise

\begin{tabular}{cccc}
\hline \hline Polarization & Bandwidth [MHz] & Azimuth resolution [m] & Swath width [km] \\
\hline Single/Dual & 84 & 7 & 350 \\
Quad & 84 & 7 & 175 \\
Single/Dual & 40 & 7 & 350 \\
Quad & 40 & 7 & 175 \\
Single/Dual & 20 & 7 & 350 \\
Quad & 20 & 7 & 175 \\
\hline \hline
\end{tabular}

Table 1: List of acquisition mode characteristics.

equivalent sigma zero better than $-25 \mathrm{~dB}$ and the ambiguity-to-signal-ratio better than $-25 \mathrm{~dB}$ in single/dual-pol mode (-22 dB in quad-pol mode). 
Acquisitions performed with a bandwidth of $84 \mathrm{MHz}$ will allow to reach a ground range resolution of $\sim 3 \mathrm{~m}$ for an incidence angle of $37^{\circ}$, required for applications like forest, ice and digital elevation models.

In Table 2 the main requirements and challenges for each research field are listed.

\begin{tabular}{ll}
\hline \hline Application & Requirements and Challenges \\
\hline Forestry & $\begin{array}{l}\text { Acquisitions with varying baselines in full-polarimetric } \\
\text { SAR interferometry performed in the morning }\end{array}$ \\
Heformation & $\begin{array}{l}\text { High number of acquisitions from different viewing geome- } \\
\text { tries }\end{array}$ \\
Agriculture & High temporal coverage especially during growing season \\
Soil Moisture & High temporal coverage with high spatial resolution \\
Global/local digital elevation models & Consistent elevation models of the whole Earth and high \\
& temporal coverage of local hot spots \\
Global land coverage & $\begin{array}{l}\text { Full-polarimetric global land mapping } \\
\text { Large baselines close to the poles, but difficult to adjust due } \\
\text { Ice dynamics }\end{array}$ \\
to formation constraints \\
Oceanography
\end{tabular}

Table 2: List of scientific requirements and challenges for each application.

The different scientific applications require different acquisitions frequencies and seasons. Acquisitions for deformation, as an example, need to be performed from different angles as often as possible. Forest structure and height acquisitions on the other hand need to be acquired with four to six different baselines during one season (maximum half a year). Acquisitions for measuring the ice dynamics need to be performed during the period of ice largest and smallest extent, i.e. during specific periods as often as possible.

A pre-study has been made in order to exploit synergies between applications, so that the same images are used for different applications, and to solve conflicts by arranging together compatible acquisitions and separate the one in conflict between ascending and descending passes. One example is the acquisition over the same area, where both forest and deformation are present. Forests need to be acquired in full polarization, which limit the possible swath width, while deformation requires only single polarization, but larger swaths (which are not available for the full-polarimatric imaging mode). In this case the acquisitions in full polarization can be used both for forest and for deformation in descending directions, while in ascending, acquisitions are planned in single polarization with the larger swath width, since no conflicts are present.

\subsection{Mission Constraints}

Besides the scientific requirements, also constraints driven by the radar instrument and by the space and ground segments have to be taken into account in the observation concept. The orbit and the instruments are designed to cover a swath width of $350 \mathrm{~km}$ on ground in single and 
dual-polarimetric mode, and $175 \mathrm{~km}$ in quad-polarimetric mode. The repeat cycle in this orbit is 16 days. Thus, each point on the Earth is seen at least 4 times in a cycle in single-polarimetric mode, although under different incidence angles and orbit directions.

The instrument also limits the possible data volume that can be acquired. For Tandem-L, as initial assumption, six ground stations are foreseen to downlink the data in Ka-Band. With these six ground stations, an estimated data volume of approximately $64 \mathrm{Tbit} / \mathrm{day}$ can be dumped. In addition, currently the on-board memory is limited to 12 Tbit at the end of life. Thus, the data volume has to be distributed properly to meet these limits.

All these different constraints are taken into account in the calculation of the acquisition timeline presented in the next chapter.

\subsection{Formation Flying}

The two SAR satellites will fly in different configurations in different operational phases to better meet all the user requirements.

In the so called constellation phase, it is possible to reduce the combined repeat-pass time of 16 days by separating the two satellites along the same orbit by a large distance. In this way it is possible to split the 16-day interval in all possible ways. At the moment, the most favorite option is so that effectively the second satellite will follow the first one after few days. This option is particular appealing for agriculture and soil moisture applications because it allows to have a weekly temporal coverage (instead of every 16 days) at the equator in one direction with the $175 \mathrm{~km}$ swath width, for repeat-pass interferometry.

In the so called bistatic phase, the two satellites will fly a controlled close helix formation [7]. This concept is inherited from the TanDEM-X mission. For Tandem-L, the formation will be based on setting slightly different inclination vectors for the orbits of the two satellites [8],[9]. In this way, the horizontal distance at the equator increases slowly over time. The drift rate is determined by the inclination difference. In such a helix formation, different baselines can be achieved. Applications like forest height and structure estimation require single-pass bistatic acquisitions with dedicated baselines. Therefore, the inclination difference is selected such that the horizontal baseline at the equator increases (or decreases) from $600 \mathrm{~m}$ up to around $20 \mathrm{~km}$ during six months as shown in Fig. 1, upper plot. In this way, suitable baselines are generated over the whole latitude range as shown for the resulting vertical wavenumber $K_{z}$ (c.f. [10]) values in Fig. 1, lower plot. The distribution of the $K_{z}$ determines the accuracy of the forest height and structure estimation. From the plot one can see that changing the baseline over one year, one can cover a wide range of $K_{z}$ values for different latitudes.

A pursuit monostatic phase is in principle also foreseen by the mission. In this case the two satellites will be separated in along track by up to $\sim 500 \mathrm{~km}$. In this configuration one can also set a cross-track and generate different baselines. This option is particularly appealing in order to obtain large baselines also at high latitudes (where it is difficult in close formation) and to reduce the transition times between different operational phases.

Considering all the factors listed above, the Tandem-L acquisition timeline is generated using a Tandem-L acquisition planning tool. It is based on the operational TanDEM-X acquisition 

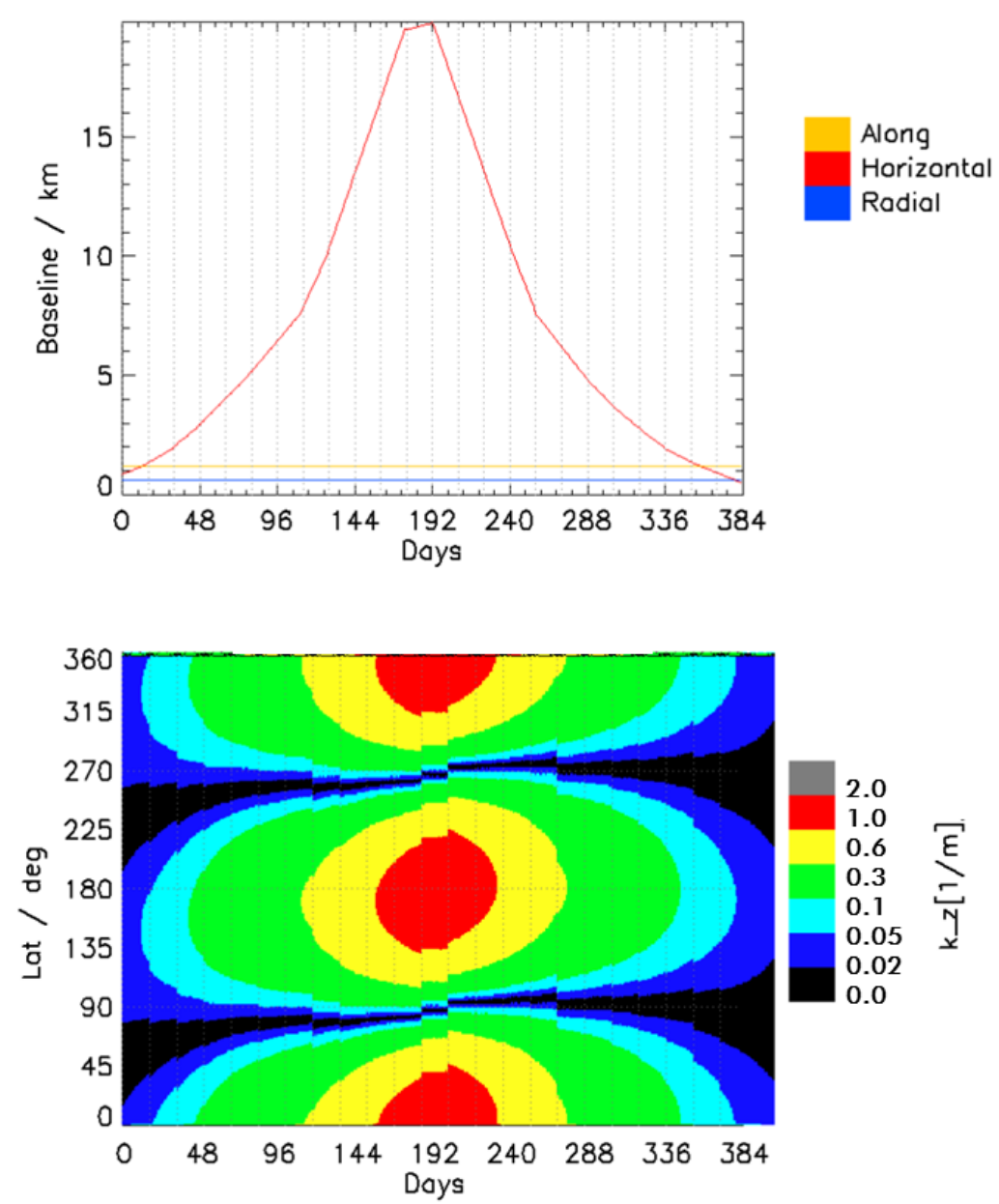

Figure 1: Tandem-L along, horizontal and radial baselines over one year (upper plot) and the respective $K_{z}$ values for different latitudes (lower plot).

planner [11] and adapted and extended for all the purposes of the new mission.

To each acquisition requirement several conditions are given: an acquisition priority, a $K_{z}$ range (in case of bistatic acquisitions), the cycles in which they have to be acquired. If all these conditions are fulfilled, then the acquisitions are planned starting with the ones with higher priorities. The systematic plan is cycle based. This means that if a certain area does not fulfill in a certain cycle the condition on the $K_{z}$, it can be acquired in another cycle. Otherwise, if a certain area is planned only for a certain cycle, if in that cycle cannot be acquired (because of conflicts with other acquisitions), it cannot be shifted to a later cycle. At the end of the generated timeline, one has to verify that the number and the temporal sampling of the acquisitions meet the initial requirements. 


\section{Acquisition Timeline Results}

As already mentioned above, the mission will operate according to different phases. Here only the results for one year of bistatic operations are presented.

The generated timeline based on the observation concept is expressed in term of acquisition times together with the information about the acquisition modes. This timeline is used to analyze the necessary system resources.

As an example of the acquisitions planned over forest can be seen in Fig. 2, where the coverage achieved in six months is shown in terms of number of acquisitions. Here one can see that all the acquisitions needed for the forest applications are planned: Six acquisitions in the tropical regions, at least five in the temperate regions and at least four in the boreal region. Because the swaths are overlapping increasing the latitude, more acquisitions are effectively planned at higher latitudes in the temperate and boreal regions.

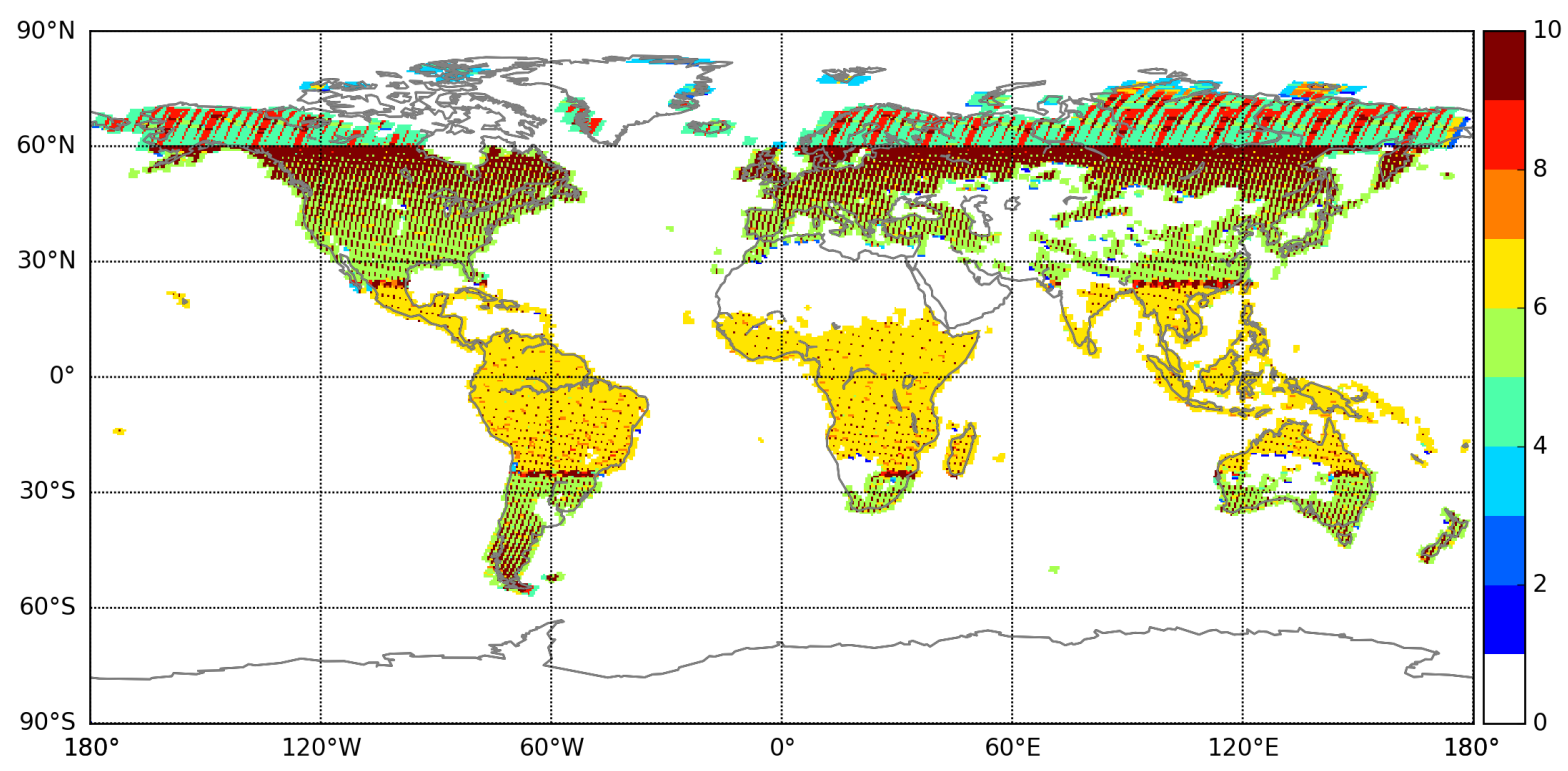

Figure 2: Number of acquisitions over the forest application areas for one season (six months)

From the timeline, data volume and orbit usage are computed and analyzed. Fig. 3 upper plot shows the mean data volume per day for the different applications for the two satellites together. As the different applications require different bandwidth, different number of polarization channels and different interferometric modes, the resulting data volume differs accordingly. For example, acquisitions over forest (in green) are acquired in single-pass interferometry with quad polarization and a bandwidth of $84 \mathrm{MHz}$. They result then in a higher data volume if compared with acquisitions for deformation (in red), which are acquired mono-statically with a single polarization and with lower bandwidth.

All these data need to be downlinked using a ground stations network with six ground stations distributed on different sites on Earth. Because the downlink in Ka-Band suffers from attenuation due to the atmospheric effects, we are currently studying the way to model a variable downlink data rate. The latter depend on the elevation of the satellite with respect to a ground 

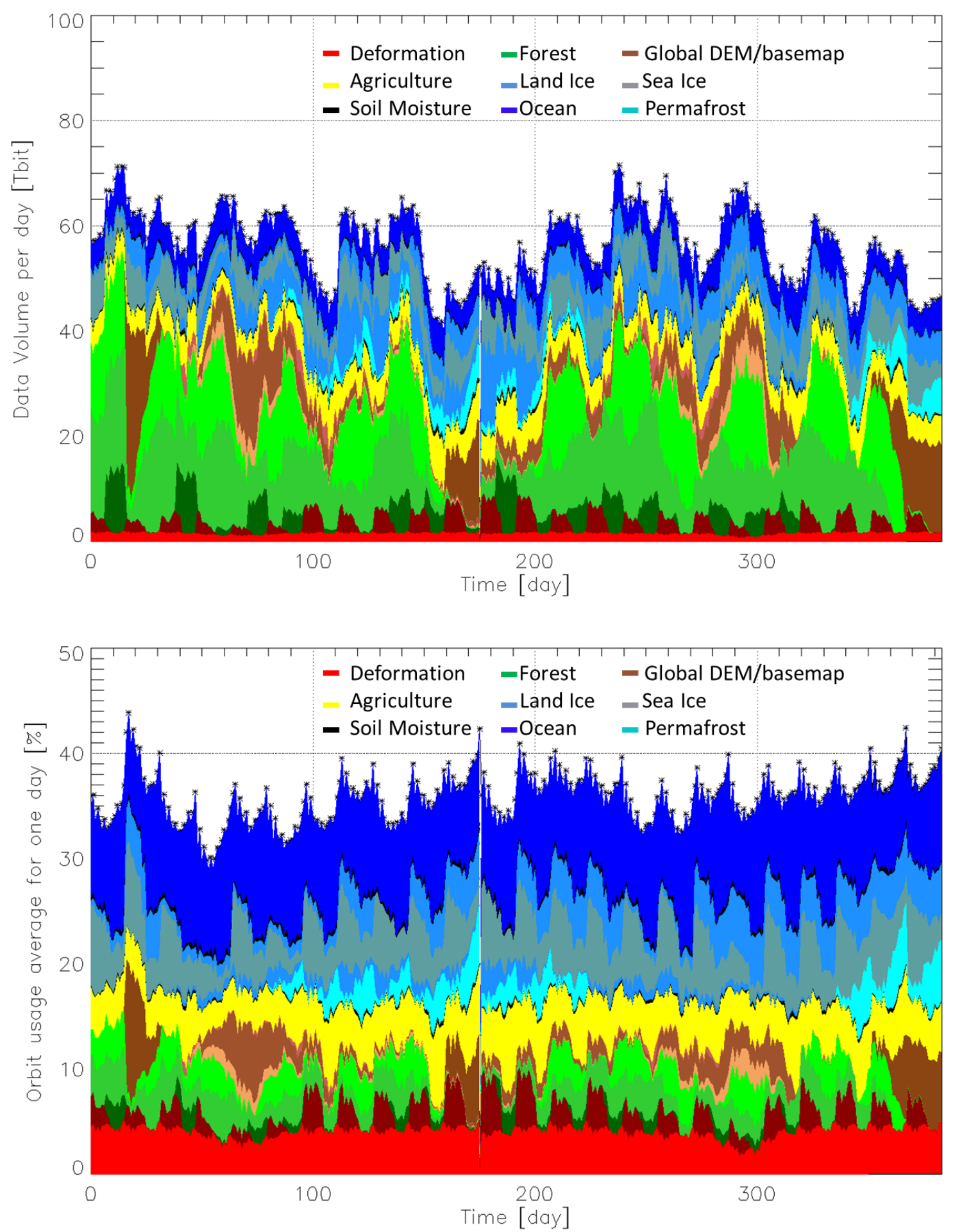

Figure 3: Daily data volume (upper plot) and mean daily orbit usage (lower plot) for the different application areas over one year. The different green colors for forest represent tropical forest (light green), temperate forest (normal green) and boreal forest (dark green). For the global DEM, dark brown color represents nominal acquisitions and light brown represents shadow/layover acquisitions from the opposite viewing geometry 
station and on the weather conditions. It will be dynamically modeled to be able to optimize the planning and downlink all the data.

Fig. 3 lower plot shows instead the mean orbit usage per day. Here one can notice that most of the orbits are used for ocean, sea ice and deformation applications. This is directly proportional to the extent of the areas which are acquired.

In order to analyze better the thermal heating of the satellites, and the power needed to support all the acquisitions, it is useful not only to know the average orbit usage along the year, but also the single orbits peaks. In Table 3, values for the peak orbits are reported. The values are given considering all the acquisitions or dividing the single-polarized acquisitions from the ones in quad polarization. Up to almost $70 \%$ of one orbit is used to acquire data. This means that less than $1 / 3$ of the orbit can be used to cool down the radar instrument.

\begin{tabular}{lllll}
\hline \hline Polarization & $\begin{array}{l}\text { Peak one orbit } \\
\text { window [\%] }\end{array}$ & $\begin{array}{l}\text { Peak three orbits } \\
\text { window [\%] }\end{array}$ & $\begin{array}{l}\text { Peak one day } \\
\text { window [\%] }\end{array}$ & $\begin{array}{l}\text { Mean } \\
\text { year [\%] }\end{array}$ \\
\hline Single & 35 & 25 & 19 & 10 \\
Quad & 58 & 46 & 37 & 27 \\
Overall & 69 & 57 & 48 & 37 \\
\hline \hline
\end{tabular}

Table 3: Orbit usages per peak units for all the single-polarization acquisitions, for all the quad-polarization acquisitions and for the total ones. Peak one orbit window is the maximum out of 5544 windows of one orbit. Peak three orbits window is the maximum out of 5544 windows of three orbits. Peak one day window is the maximum out of 5544 windows of one day (15 orbits). Mean year is the average over 384 days, corresponding to 24 orbit cycles.

\section{Conclusion}

In this paper we have shown an observation concept developed for the Tandem-L mission. Based on the scientific requirements, on the constraints of the radar instrument, and on the formation flying, a feasible acquisition timeline has been generated. The resulting timeline fulfills all requirements for the various scientific applications. Daily data volumes reach up to more than 64 Tbit and the peak orbit usage is close to 70\%. If all data can be downlinked to the currently foreseen network of Ka-Band ground stations is to be confirmed by the end of the Phase B study.

In the following project phases, this observation concept will be further refined and validated along with the performance estimation and predicted accuracy of the information products foreseen for Tandem-L.

\section{References}

[1] A. Moreira, G. Krieger, I. Hajnsek, K. Pa-pathanassiou, M. Younis, P. Lopez-Dekker, S. Huber, M. Villano, M. Pardini, M. Eineder, F. De Zan, and A. Parizzi, "Tandem-L: A Highly Innovative Bistatic SAR Mission for Global Observation of Dynamic Processes on the Earth's Surface," IEEE Geoscience and Remote Sensing Magazine, vol. 3, no. 2, pp. 8-23, 2015. 
[2] S. Huber, M. Younis, A. Patyuchenko, G. Krieger, and A. Moreira, "Spaceborne reflector SAR systems with digital beamforming," IEEE Transactions on Aerospace Electronic System, vol. 48, pp. 3473-3493, 2012.

[3] M. Villano, G. Krieger, and A. Moreira, "taggered SAR: high resolution wide-swath imaging by continuous PRI variation," IEEE Transactions on Geoscience and Remote Sensing, vol. 52, pp. 4462-4479, 2014.

[4] M. Bachmann, D. Borla Tridon, F. De Zan, G. Krieger, and M. Zink, "Tandem-L observation concept an acquisition scenario for the global scientific mapping machine," in European Conference on Synthetic Aperture Radar (EUSAR), 2016.

[5] M. Zink, M. Bachmann, B. Bräutigam, T. Fritz, I. Hajnsek, A. Moreira, B. Wessel, and G. Krieger, "The New Global DEM Takes Shape," IEEE Geoscience and Remote Sensing Magazine, vol. 2, no. 2, pp. 8-23, 2014.

[6] M. Zink, M.Bachmann, B. Bräutigam, T. Fritz, I. Hajnsek, G. Krieger, A. Moreira, and B. Wessel, TanDEM-X: Das neue globale Höhenmodell der Erde. Springer, 2016.

[7] R. Kahle, H. Runge, J.-S. Ardaens, S. Suchandt, and R. Romeiser, "Formation Flying for AlongTrack Interferometric Oceanography - First In-Flight Demonstration with TanDEM-X," Acta Astronautica, vol. 99, pp. 130-142, 2014.

[8] H. Fiedler and G. Krieger, "Close Formation Flight of Passive Receiving Micro-Satellites," in International Symposium On Space Flight Dynamics, 2004.

[9] S. D'Amico and O. Montenbruck, "Proximity Operations of Formation-Flying Spacecraft Using an Eccentricity/Inclination Vector Separation," Journal of Guidance, Control, and Dynamics, vol. 29, no. 3, pp. 554-563, 2006.

[10] M. Pardini, A. Torano-Caicoya, F. Kugler, and K. Papathanassiou, "Estimating and understanding vertical structure of forests from multibaseline TanDEM-X Pol-InSAR data," in IEEE International Geoscience and Remote Sensing Symposium (IGARSS), 2015.

[11] C. Ortega-Miguez, D. Schulze, M. Polimeni, J. Böer, P. Rizzoli, and M. Bachmann, "TanDEM-X Acquisition Planner," in European Conference on Synthetic Aperture Radar (EUSAR), 2012. 